\title{
Visión prospectiva para las Mipymes del subsector artesanal en Pasto
}

\author{
Germán Alfredo López Montezuma' \\ Diego Germán Martínez Delgado²
}

\section{Resumen}

Este trabajo resume las condiciones actuales de la actividad artesanal en Colombia, enmarcada desde la región que lidera la actividad en el sur del país, con el fin de estructurar un panorama real y visualizar la actividad artesanal como empresa, con proyección de su mercado, en busca del mejoramiento de un aporte a la economía colombiana, como alternativa de empleo y de desarrollo de los artesanos.

Por lo anterior, se realizará un plan prospectivo para los artesanos del municipio de Pasto, como herramienta para alcanzar mayor nivel de competitividad, a partir de la identificación de los aspectos internos y externos que afectan el sector en el cumplimiento de su objetivo.

El objetivo general de este proyecto es establecer los escenarios futuros para la administración de MIPYMES del subsector de las artesanías en Pasto. Se busca realizar un análisis de los modelos tradicionales de administración existentes, una prospección de los escenarios deseados para la administración de MIPYMES, con un enfoque alternativo, en pro de generar bienestar empresarial y diseñar un plan de acción para establecer el proceso lógico de implementación del escenario deseado.

Palabras clave: Prospectiva, artesanías, barniz de Pasto, escenario futuro, asociatividad, modelo básico.

\section{Abstract}

This work summarizes the current conditions of the handmade activity in Colombia, framed from the region that leads the activity, south of the country, with the purpose of to structure a real panorama and to visualize the handmade activity as company, with projection of its market in search from a contribution to the Colombian economy as employment alternative and the artisans' development. Reason why he/she will be carried out a prospective plan for the artisans of the municipality of Grass, as tool to reach greater level of competitiveness, starting from the identification of the internal and external aspects that you/they affect to the sector in the execution of their objective.

The overall objective of this project is to "establish the future scenarios for the administration of the Msmes subsector of the handicrafts in grass," where we intended to perform an analysis of the traditional models of existing management for the Msmes in Pasto, A prospecting of the desired scenarios for the administration of Msmes, under an alternative approach for the generation of welfare, and design a plan of action to establish the logical process of implementation of the desired scenario.

Key words: Prospective, crafts, barniz de Pasto, future scenario, associatively, basic model

Recibido: 16 de abril de 2012

Aceptado: 27 de julio de 2012

\footnotetext{
${ }^{1}$ Magister en docencia universitaria. Especialista en finanzas. Administrador financiero. Docente auxiliar, ECACEN Pasto UNAD. german. lopez@unad.edu.co,grupo de investigación Sindamanoy

${ }^{2}$ Magister en mercadeo agroindustrial. Especialista en proyectos de desarrollo. Economista. Docente ocasional, ECACEN Pasto UNAD. diego. martinez@unad.edu.co,grupo de investigación Sindamanoy
} 


\section{Introducción}

Las actividades artesanales son poco valoradas: incluso, los mismos artesanos deben buscar alternativas diferentes de subsistencia, y dejar a un lado aquello que los hace únicos. Es el momento de buscar el protagonismo para esa actividad, aprovechando las nuevas tendencias organizacionales y la globalización de los mercados, para hacer de la actividad artesanal una muestra representativa de cultura, originalidad y aporte alternativo a la economía, e impedir que se pierdan las costumbres milenarias de los antepasados y se las convierta en la fuente de recursos que ayuden a fortalecer las economías locales.

El departamento de Nariño se ha caracterizado en todo el país por ser una región tradicionalmente artesanal, donde se elaboran piezas artesanales con técnicas milenarias indígenas, como el enchapado en tamo y la aplicación del barniz de Pasto, o MopaMopa, que es la hueIla dactilar que identifica a una raza pujante y artística.

Desafortunadamente, esta actividad, como actividad económica, no ha brindado al sector la fortaleza suficiente para convertir a la región del suroccidente colombiano en una potencia que pueda económicamente solventar, por lo menos, la subsistencia del artesano. A pesar de que hay entidades que, aparentemente, brindan el apoyo de capacitación a los artesanos, se puede fácilmente comprobar en la región que existe un estancamiento comercial de los productos artesanales elaborados y decorados con técnicas ancestrales.
Se hace necesario trabajar en la búsqueda de escenarios prospectivos con los cuales se pueda visualizar un sector más competitivo en la ciudad de Pasto, de tal manera que se logre su reconocimiento a escala nacional e internacional, por su calidad, la tradición de sus productos y su organización empresarial.

En este orden de ideas, el presente trabajo se desarrolla con el objetivo principal de diseñar un plan prospectivo para los artesanos del barniz de Pasto según la técnica de enchapado en tamo, como una técnica que genere competitividad y mayor participación en el mercado.

\section{Métodos}

Para el desarrollo del proyecto se escogió el tipo de investigación etnográfico, pues se busca una descripción profunda de las características de un grupo humano específico que comparte intereses, ideales y patrones culturales similares: es decir, una investigación que demuestre las apreciaciones de los artesanos de la ciudad de Pasto, a partir del sentido y el significado hacia la construcción de comunidad, lo cual se constituye en el objeto de estudio de la presente investigación.

La exigencia de la investigación motivó la utilización del paradigma mixto (cuantitativo-cualitativo), por cuanto se analizaron comportamientos presentados por los actores del subsector y se los combinó con los datos estadísticos, para facilitar la tendencia prospectiva.

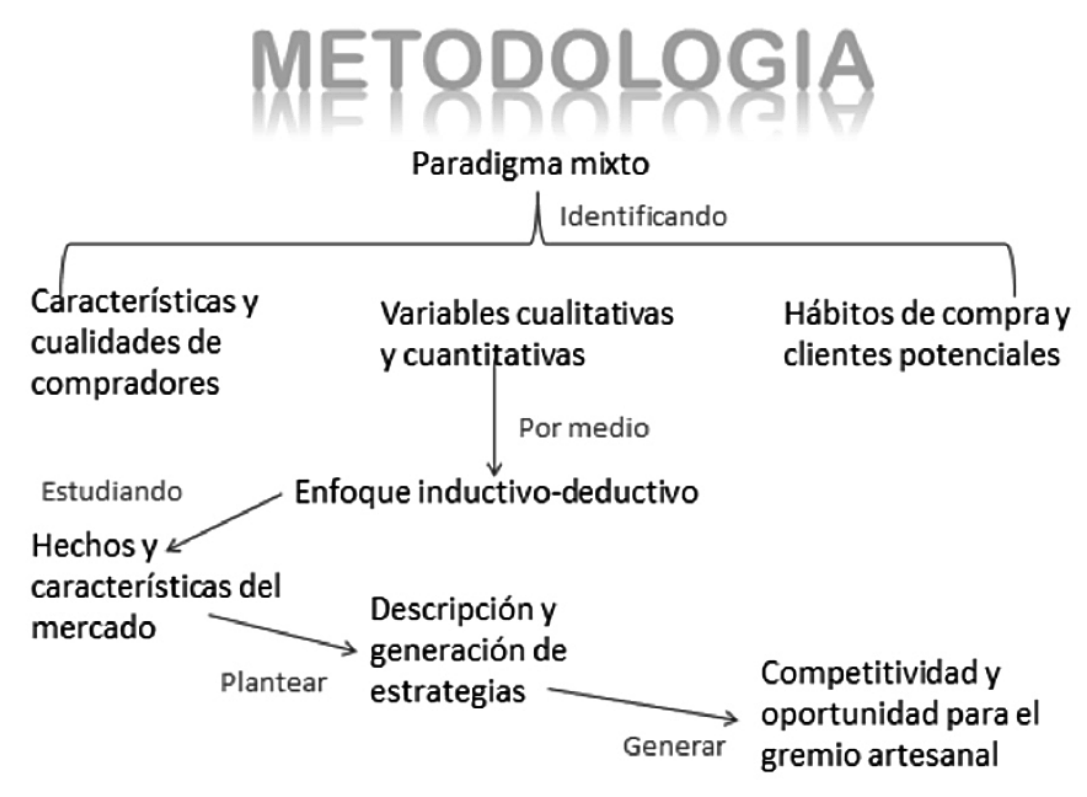

Gráfico 1. Metodología de investigación 
Dentro del proceso sistémico de la investigación, la metodología de estudio se enmarca desde un enfoque inductivo-deductivo. Se parte de unos hechos particulares que caracterizan a los actores, para luego proceder a la descripción, y a reconocer e identificar formas de conducta actitudinales y comportamentales de los artesanos.

Para obtener la información primaria se trabajó siguiendo el modelo básico Godetiano de la escuela voluntarista, adaptado para Latinoamérica por Francisco José Mójica (2008). Se tomó como fuente primaria el taller de expertos, donde un grupo de especialistas reconocidos en los campos referentes al sector de las artesanías se reunieron y emitieron juicios y conceptos sobre seis puntos específicos de la investigación, y que son: Situa- ción actual de la empresa artesanal: Políticas de apoyo a la organización de las empresas artesanales; Comportamiento del mercado (interno y externo); Estructura organizativa de las empresas; Tendencias organizacionales en el sector artesanal; y Crédito de Fomento.

Las fuentes secundarias de la información fueron el estado del arte y las tendencias mundiales sobre factores de cambio y variables estratégicas, lo cual permitió plantear los escenarios prospectivos del sector artesanal del barniz de Pasto. Como bien lo manifiesta Francisco Mójica (2008), el estado del arte permite conocer cómo es hoy, y cómo era en el pasado, la organización. Fue un análisis basado en datos tanto cualitativos como cuantitativos, que respaldan las condiciones actuales e históricas del sector artesanal de Pasto.

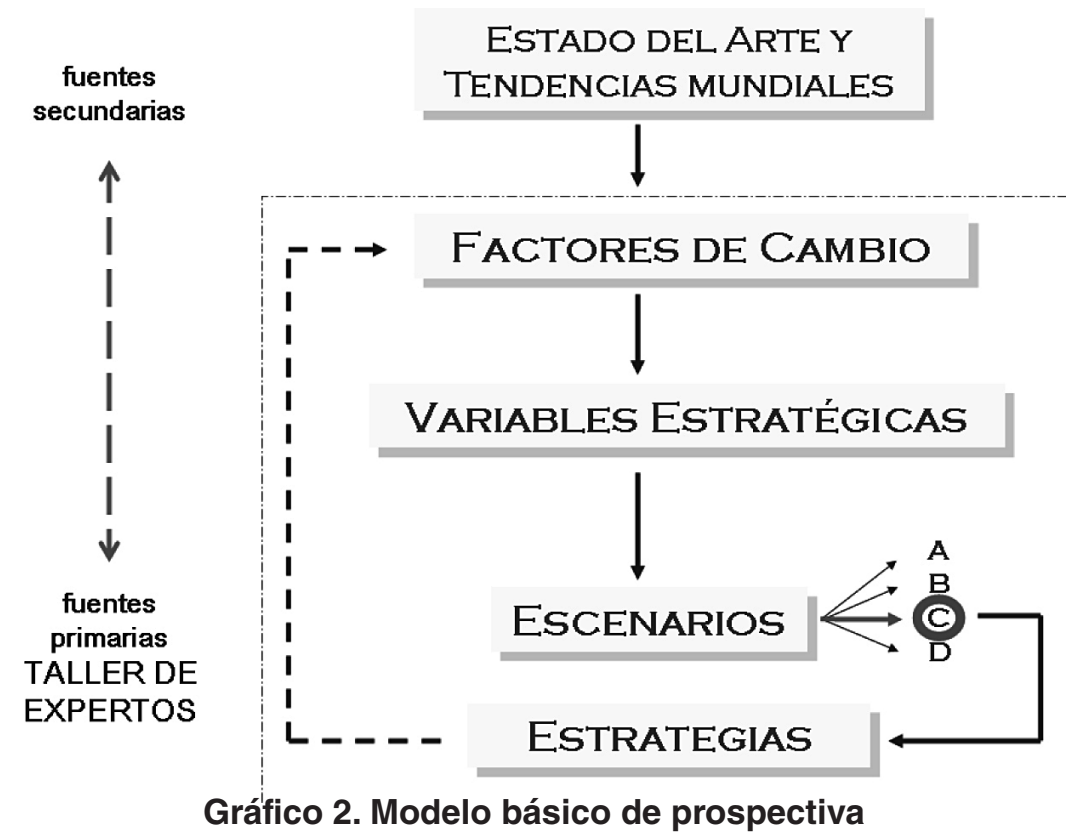

Por medio de las herramientas Lipsor, se diseñaron talleres de prospectiva con la presencia de los cuatro actores sociales (Estado, sector artesanal, academia y sociedad civil), quienes fueron los artífices de la construcción de la matriz DOFA analizando los aspectos endógenos y exógenos del sector.

Una vez realizado el diagnóstico del subsector se procedió a utilizar la primera herramienta: la matriz de impacto cruzado; ello permitió definir las variables evaluadas desde su dependencia y su influencia. La segunda herramienta prospectiva fue la aplicación MACTOR, donde se definieron los actores, su posición estratégica actual y su comportamiento futuro. Como herramienta de diseño de escenarios futuros se utilizó el sistema de matriz de impactos cruzados (SMIC), que permitió trabajar cinco hipótesis y medir su probabilidad.

\section{Discusión y resultados}

Uno de los objetivos del presente trabajo es presentar los resultados de una investigación adelantada en el subsector artesanal del municipio de Pasto, teniendo en cuenta las políticas gubernamentales de inclusión productiva y el Plan de Competitividad para Nariño, el cual se convierte en la carta de navegación para promover el desarrollo económico y mejorar la calidad de vida de la región, con el compromiso y la concertación de los diferentes actores públicos y privados (PRCN 2008-2011).

\section{Entorno económico}

De acuerdo con el Censo Económico Nacional sobre el sector artesanal, realizado por Artesanías de Colombia (1992-1994), 260000 personas producen artesanías, lo cual representa el $15 \%$ de la población ocupada de la industria manufacturera. De este porcentaje total, la 
mayor concentración se ubica en los departamentos de Nariño $(14,34 \%)$, Sucre $(10,06 \%)$, Córdoba $(9,34 \%)$, Boyacá $(8,43 \%)$, Cesar (6,95\%), Atlántico $(6,52 \%)$ y Tolima (5,15\%).

Los oficios más importantes y con un mayor número de artesanos dedicados a estas actividades son la tejeduría, con el $57,52 \%$, la madera, con el $13,48 \%$, y la cerámica, con el $7,37 \%$.

Las condiciones de marginalidad económica y social del sector se reflejan en los bajos niveles de escolaridad. Un $17 \%$ de la población es analfabeta; cifra superior al promedio nacional, que está por debajo del $5 \%$. Del $52 \%$ de quienes asistieron a la Primaria, tan solo el $18 \%$ la terminaron. Un $26 \%$ inició estudios secundarios, y de ellos los culminaron el $8 \%$, y tan solo un $3 \%$ accedió a la educación superior técnica universitaria.

Los bajos niveles de escolaridad se relacionan con el hecho de que el aprendizaje de los oficios artesanales es de carácter informal, y se da en el contexto familiar y por iniciativa personal, mediante la participación directa en los procesos productivos. El carácter de su aprendizaje es altamente informal, y se concentra en el aspecto técnico del proceso y en el formal del producto, donde juega un papel preponderante la intuición, potenciada, a su vez, por el ejercicio cotidiano del trabajo. El 48,32\% aprende el oficio en su hogar; el 14,39\%, en talleres particulares como aprendiz; y por un sistema de cursos de capacitación en oficios, únicamente el 7,32\%.

El $56,11 \%$ del sector artesanal desarrolla su actividad en pequeñas unidades productivas y de forma individual. Algunos de los problemas que afrontan estos talleres son: dificultades por escasez de mano de obra $(21,33 \%)$, inestabilidad $(10,89 \%)$ y falta de personal calificado (8,96\%).

En lo que respecta a niveles y grados de organización, el censo reveló que el $82,38 \%$ de los artesanos no ha participado en ningún tipo de organización, y que solo el $12,81 \%$ pertenece a organizaciones gremiales, comunitarias y para la producción y la comercialización.

La mayor parte de la producción artesanal se vende en los talleres o viviendas. Un $0,30 \%$ se vende en plaza de mercado, y únicamente el $11,58 \%$ vende en otros sitios; el $0,03 \%$ de los artesanos participa en ferias artesanales, y el 0,01\%, de forma ambulante. De igual manera, el $85,16 \%$ de la producción se vende en los municipios de origen: el $8,18 \%$, en otros municipios, y solamente el $3,45 \%$, en otros departamentos, lo que explica las grandes debilidades existentes en los procesos de comercialización.

\section{Matriz DOFA}

\section{Análisis exógeno}

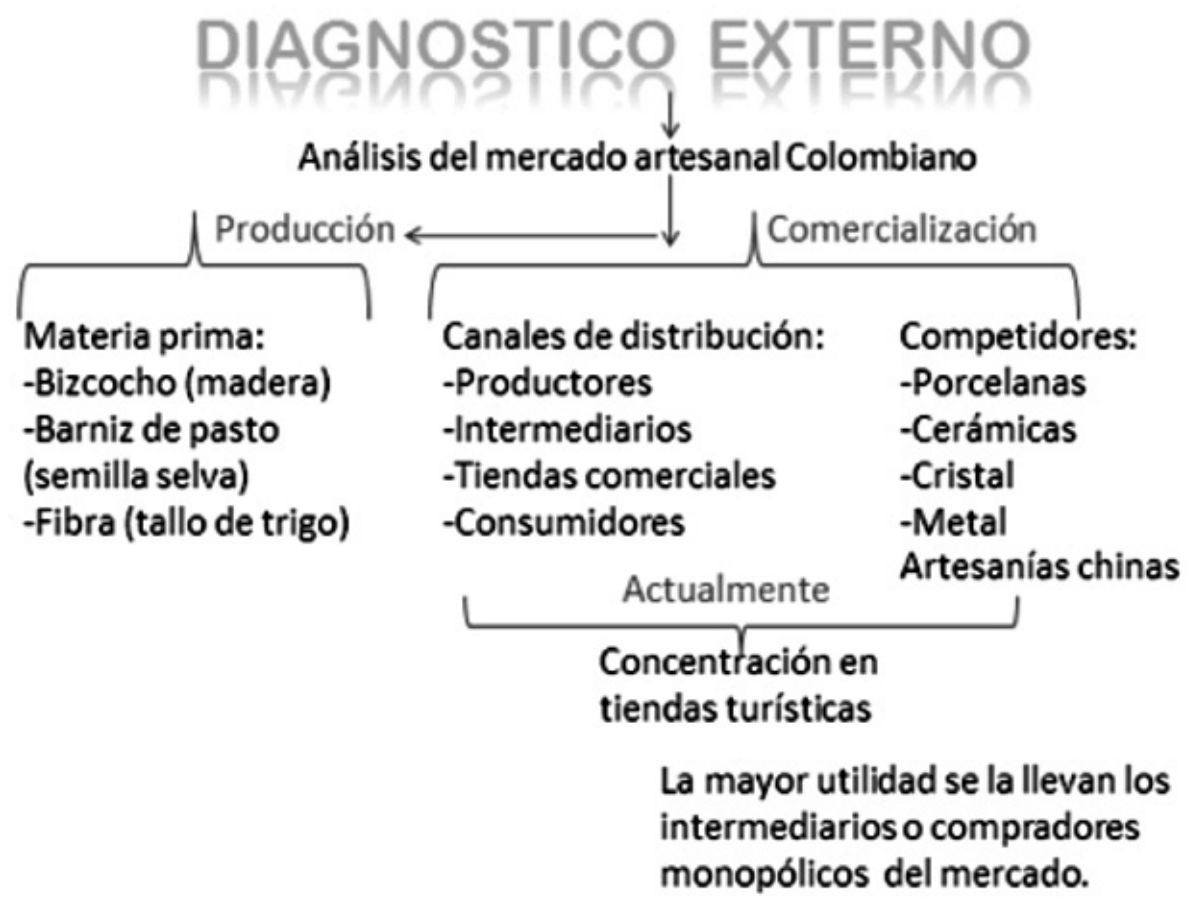

Gráfico 3. Diagnóstico externo 


\section{Oportunidades}

- La artesanía colombiana es cada vez más reconocida en el mundo entero, lo que le ha permitido una buena aceptación en el mercado internacional.

- Con base en los diseños tradicionales, los artesanos colombianos desarrollan nuevas líneas en sus productos, acordes a las tendencias en el mercado exterior, lo que aumenta la potencialidad de este sector.

- La artesanía colombiana es rica en productos, temas y coloridos que satisfacen gustos variados.

- La tradición cultural brinda el toque de originalidad a los productos y garantiza su calidad de elaboración a través de un conocimiento transmitido de generación en generación desde hace muchos años.

- La tradición artesanal y la adecuada capacitación en el sector proveen mano de obra de calidad.

- Los artesanos adaptan a sus productos tradicionales nuevas tendencias del gusto internacional.

- Realización de ferias, turismo y ruedas de negocios que permiten el contacto con intermediarios.
- El alto crecimiento del sector turismo en el país permite comercializar el producto de forma directa con el consumidor.

- Posicionarse en el mercado nacional como la primera potencia artesanal del país.

- Organización de la actividad según parámetros legales, para obtener beneficios gubernamentales.

\section{Amenazas}

- La competitividad de la calidad y los costos de los productos.

- Competencia con países asiáticos que cuentan con mayor nivel tecnológico y alta producción ofreciendo un costo menor al consumidor.

- Difícil acceso a mercados internacionales.

- Condiciones de pobreza extrema en un alto porcentaje de la población artesanal.

- Pérdida permanente de la tradición cultural y artística que identifica la actividad en el sur del país.

\section{Análisis endógeno}

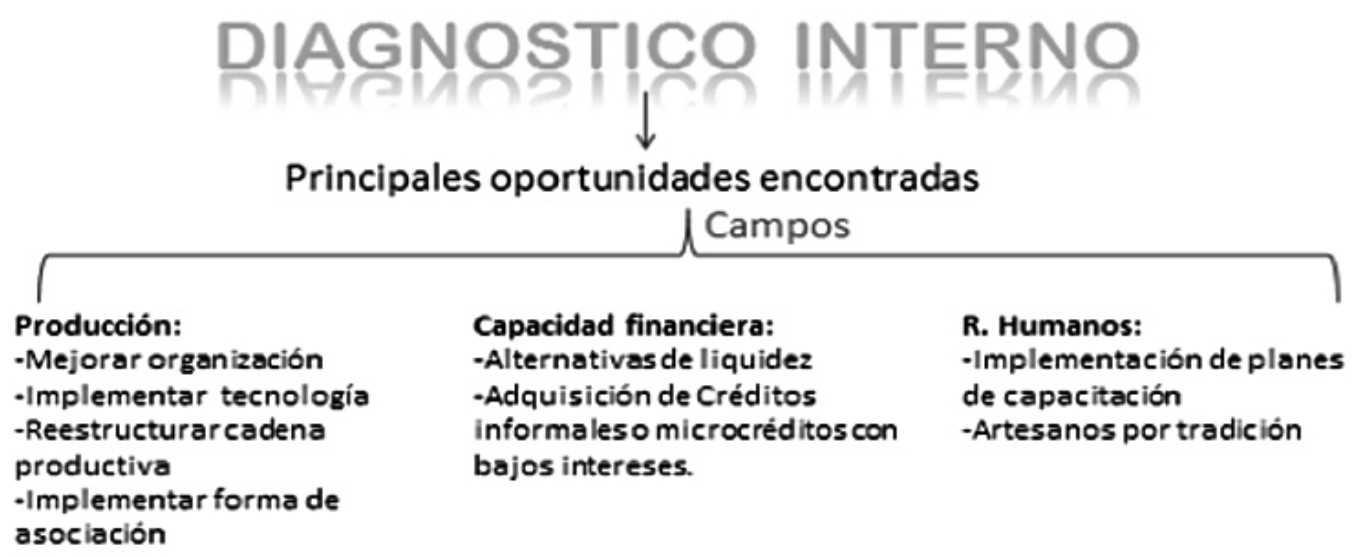

Inv. Y desarrollo:

- Mejorar la formación académicay

cultural.

- Mejora en la imagen y generar un

amplio portafolio de productos.
Comercialización:

- Mayor participación en ferias.

- Venta en municipios de origen

- Venta en hogares

-Venta en plazas de mercado

Gráfico 4. Diagnóstico interno 


\section{Fortalezas y debilidades del proceso admi- nistrativo del sector}

\section{Fortalezas}

- Dada la importante participación que tienen los artesanos del departamento de Nariño (14\%) del total de artesanos del país, el Gobierno Nacional, por medio del Ministerio de Comercio, industria y Turismo, se ha preocupado durante los últimos años por fortalecer, capacitar y apoyar al gremio de artesanos, para que siga siendo una de las principales industrias generadoras de empleo en el sector de la industria manufacturera en Colombia.

- Reconocimiento y amplia trayectoria de la artesanía Nariñense.

- Recurso humano calificado, con conocimiento y experiencia de las técnicas artesanales.

\section{Debilidades}

- Un alto porcentaje de los artesanos del departamento de Nariño han adquirido su conocimiento empíricamente, por legados y enseñanzas de sus familias, lo cual, en la gran mayoría de los casos, no les ha permitido una formación académica adecuada. Ante esto, los diferentes procesos productivos, administrativos y de comercialización aún no son bien desarrollados, y ello genera falencias que muchas veces no les permiten construir una industria rentable y sostenible en el tiempo.
- Falta de conocimiento del mercado nacional.

- Falta de infraestructura.

- Falta de recursos necesarios: financieros, tecnológicos, de sistemas, de logística.

- Falta de estatus legal y de reconocimiento jurídico.

\section{Análisis prospectivo}

Para realizar el análisis prospectivo, como lo manifiesta Godet (2008), no existe un método único de escenarios; sin embargo, a continuación se describen las acciones realizadas dentro del proceso investigativo, como el examen sistémico de los futuros posibles.

\section{Determinación de variables clave}

Como resultado del diagnóstico y del trabajo con expertos fue posible identificar 20 variables clave; ellas son: iniciativa empresarial, comercialización, promoción, mano de obra, miedo al cambio, competencia, contrabando, centro de convenciones empresariales, malas experiencias, continuidad, resultados rápidos, falta de patrocinio, tradicionalismo, conformismo, falta de dedicación, protección social, estructura organizacional, empirismo, individualismo y asesorías externas.

La motricidad y dependencia del conjunto de las variables de las filas y las columnas se observa en la siguiente tabla

Tabla 1. Matriz de Influencias Directas (MID)

\begin{tabular}{|c|c|c|c|c|c|c|c|c|c|c|c|c|c|c|c|c|c|c|c|}
\hline & $\begin{array}{l}\vec{n} \\
\text { 离 } \\
\end{array}$ & \begin{tabular}{l}
$N$ \\
\hdashline \\
$\varrho$ \\
3
\end{tabular} & $\begin{array}{l}\omega \\
\ddot{0} \\
\text { Do } \\
\text { ○े }\end{array}$ & $\begin{array}{l}n \\
z \\
0\end{array}$ & 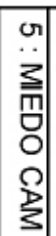 & 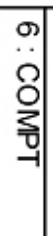 & 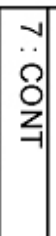 & 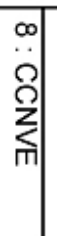 & & $\begin{array}{l}\overrightarrow{0} \\
\ddot{\jmath} \\
\text { ఏે } \\
\text { ż }\end{array}$ & $\begin{array}{l}\overrightarrow{\vec{a}} \\
\ddot{\bar{z}} \\
\mathbf{3}\end{array}$ & 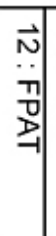 & $\begin{array}{l}\vec{\omega} \\
\ddot{\partial} \\
\overrightarrow{0} \\
\vec{b}\end{array}$ & & & & & $\begin{array}{l}\overrightarrow{0} \\
\ddot{z} \\
0\end{array}$ & 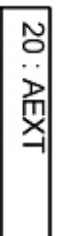 \\
\hline $1:$ IEM & 0 & 2 & 0 & 0 & 0 & 2 & 1 & 1 & 2 & 2 & 0 & 1 & 1 & 1 & 1 & 2 & 1 & 1 & 3 \\
\hline $2: \mathrm{COM}$ & 1 & 0 & 3 & 0 & 0 & 3 & 1 & $\mathrm{P}$ & 0 & 2 & 1 & 2 & 1 & 0 & 0 & 1 & 0 & 0 & 2 \\
\hline 3: PROM & 2 & 3 & 0 & 1 & 2 & 3 & 3 & $\mathrm{P}$ & 1 & 2 & 1 & 3 & 1 & 1 & 1 & 1 & 1 & 1 & 3 \\
\hline $4: \mathrm{MO}$ & 1 & 2 & 2 & 0 & 1 & 1 & 1 & 1 & 0 & 3 & 1 & 1 & 2 & 1 & 1 & 3 & 1 & 2 & 3 \\
\hline 5: MIEDO CAM & 3 & 3 & 3 & 0 & 0 & 2 & 1 & 0 & 3 & 1 & 2 & 1 & 1 & 1 & 3 & 1 & 2 & 2 & 3 \\
\hline $6:$ COMPT & 3 & 3 & 3 & 2 & 2 & 0 & 3 & 1 & 1 & 3 & 1 & 3 & 1 & 1 & 2 & 1 & 2 & 2 & 3 \\
\hline $7:$ CONT & 1 & 3 & 3 & 1 & 0 & 3 & 0 & $\mathrm{P}$ & 0 & 0 & 0 & 1 & 1 & 1 & 1 & 2 & 1 & 1 & 0 \\
\hline $8:$ CCNVE & 2 & $\mathrm{P}$ & 3 & 3 & 2 & 3 & 2 & 0 & 1 & 3 & 1 & 2 & 3 & 3 & 3 & 2 & 2 & 2 & 3 \\
\hline $9:$ MEX & 1 & 2 & 2 & 2 & 3 & 2 & 1 & 0 & 0 & 1 & 1 & 1 & 1 & 1 & 2 & 0 & 2 & 3 & 3 \\
\hline $10:$ CONTI & 3 & 3 & 3 & 2 & 2 & 3 & 1 & $P$ & 1 & 0 & 1 & 2 & 1 & 1 & 1 & 3 & 2 & 3 & 3 \\
\hline 11 : INME & 1 & 3 & 3 & 3 & 3 & 3 & 1 & $P$ & 2 & 1 & 0 & 2 & 2 & 2 & 2 & 1 & 3 & \begin{tabular}{l|l}
3 \\
\end{tabular} & 3 \\
\hline $12:$ FPAT & 3 & 3 & 3 & 2 & 3 & 3 & 3 & $\mathrm{P}$ & 2 & 2 & 1 & 0 & 2 & 3 & 3 & 2 & 2 & 2 & 3 \\
\hline $13:$ TRAD & 1 & 1 & 1 & 1 & 3 & 2 & 1 & 1 & 2 & 2 & 3 & 3 & 0 & 3 & 3 & 1 & 3 & 3 & 2 \\
\hline $14:$ CONF & 1 & 1 & 1 & 2 & 3 & 2 & 2 & 1 & 2 & 1 & 3 & 3 & 3 & 0 & 3 & 1 & 3 & 3 & 2 \\
\hline $15:$ FDED & 1 & 1 & 1 & 2 & 3 & 3 & 2 & 1 & 3 & 2 & 2 & 2 & 2 & 3 & 0 & 3 & 3 & 3 & 3 \\
\hline $16:$ PSOC & 3 & 1 & 1 & 3 & 1 & 1 & 1 & 1 & 1 & 2 & 1 & 2 & 2 & 2 & 2 & 3 & 2 & 2 & 2 \\
\hline $17:$ EOR & 3 & 3 & 3 & 3 & 3 & 3 & 2 & 3 & 1 & 3 & 1 & 1 & 2 & 2 & 2 & 0 & 2 & 2 & 3 \\
\hline $18:$ EMP & 1 & 1 & 1 & 2 & 2 & 2 & 1 & 0 & 3 & 1 & 3 & 3 & 3 & 3 & 3 & 1 & 0 & 3 & 1 \\
\hline $19:$ IND & 1 & 1 & 1 & 1 & 2 & 1 & 1 & 0 & 3 & 2 & 3 & 3 & 3 & 3 & 3 & 1 & 2 & 0 & 1 \\
\hline $20:$ AEXT & 3 & 3 & 3 & 3 & 3 & 3 & 2 & \begin{tabular}{l|l}
$P$ \\
\end{tabular} & & 3 & 1 & 1 & 1 & & 1 & 3 & 2 & \begin{tabular}{|l|l}
3 \\
\end{tabular} & \begin{tabular}{|l|}
0 \\
\end{tabular} \\
\hline
\end{tabular}




\section{Eje de la estrategia}

Con el fin de conocer las variables estratégicas, luego del análisis estructural es posible identificar las varia-

bles con mayor motricidad y dependencia, representadas en el siguiente gráfico:

Plano de influencias / dependencias directas

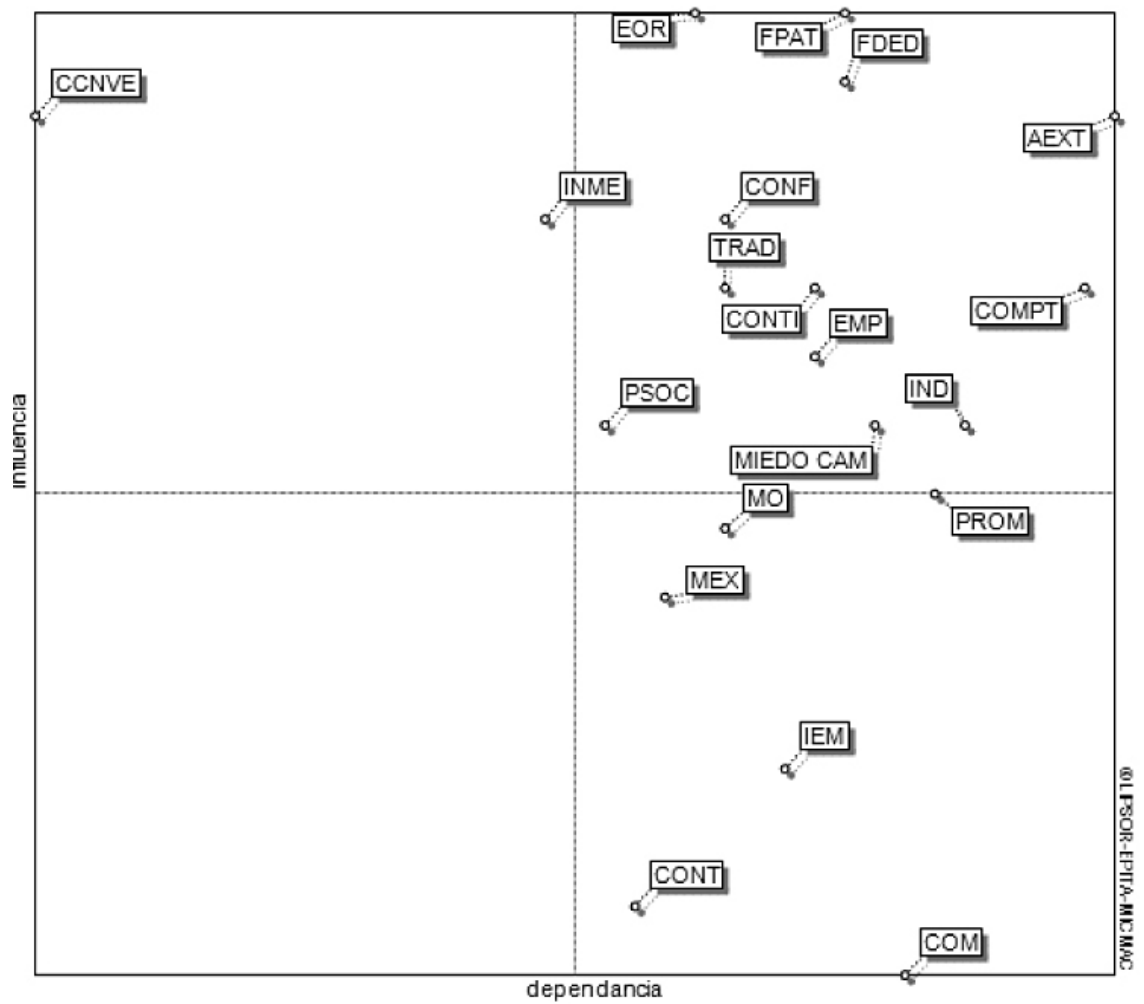

Gráfico 5. Plano de motricidad y dependencia

Teniendo en cuenta que el eje de la estrategia es un grupo de variables sobre una línea imaginaria que parte desde el vértice inferior izquierdo y se desplaza hasta el vértice superior derecho del plano, donde se sitúan las variables clave, es posible conocer cuáles son los retos estratégicos para el subsector de las artesanías.

Según el criterio de la motricidad con el grado de dependencia (Godet 2009), la comparación de la jerarquía de las variables en las diferentes clasificaciones (directa, indirecta y potencial) permite confirmar la importancia de ciertas variables, así como descubrir variables que, por su acción indirecta, desempeñan un papel preponderante aunque no hayan sido detectadas por la clasificación directa. Se identificaron: Protección social, Tradicionalismo, Conformismo, Asesorías externas, Competencia, Individualismo, Miedo al cambio, Continuidad y Empirismo.

\section{Técnica de juego de actores}

En el método de análisis de juego de actores, Mactor busca valorar las relaciones de fuerza entre los actores y estudiar sus convergencias y divergencias respecto a un cierto número de posturas y de objetivos asociados (Godet M., 2007).

Con base en el concepto anterior, se identificaron los actores que intervienen, y con ellos se definieron los intereses que dinamizan actuar de una u otra manera, pues al final son ellos los que intervienen para que los escenarios se concreten o no. Los actores identificados son: Red UREL (Red de Universidades Regionales Latinoamericanas), Laboratorio de Artesanos Pasto (LABART), ZEIKY (Centro de información y asesoría en comercio exterior), CORPOCARNAVAL (CORPO), Medios de comunicación (MCOM), Representante de los artesanos (REPART).

Como se puede observar en la grafica, se busca valorar las relaciones de fuerza entre los actores y estudiar sus convergencias y divergencias con respecto a un cierto número de actitudes y de objetivos relacionados. 
Map of influences and dependences between actors

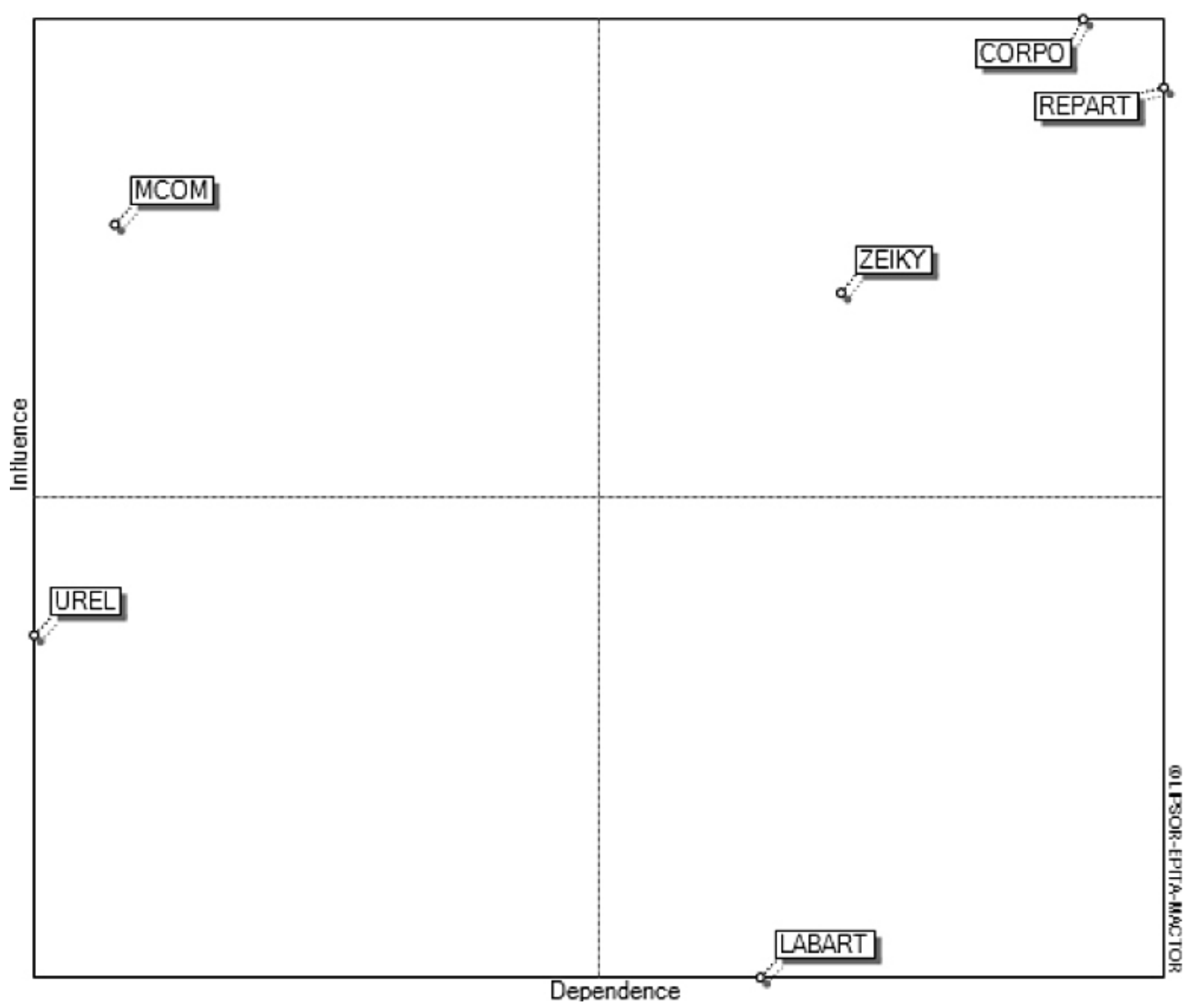

Gráfico 6. Plano de influencias y dependencias entre actores

\section{Técnica de escenarios}

Teniendo en cuenta que un escenario es el instrumento de planificación que permite medir y elegir alternativas de desarrollo, se constituye en un proceso de aprendizaje donde es posible combinar datos cualitativos o cuantitativos.

Los métodos de impactos cruzados probabilistas permiten determinar las probabilidades simples $y$ condicionadas de eventos o hipótesis, así como las probabilidades de combinaciones de estos últimos, teniendo en cuenta las interacciones entre los eventos o las hipótesis (Godet, M., 2007).
El objetivo de estos métodos no es solo hacer destacar los escenarios más probables, sino, también, examinar las combinaciones de hipótesis que serán excluidas a priori (Godet, M., 2007).

Con las variables claves definidas en el estudio se procedió con el grupo de expertos a aplicar la técnica del SMIC (Sistema de Matrices de Impacto Cruzados), con el fin de conocer la probabilidad de ocurrencia de los escenarios y el tipo de tendencia que cada uno de los eventos presenta. 
Tabla 2. Cuadro de escenarios probables

\begin{tabular}{|c|c|c|c|}
\hline \multicolumn{2}{|r|}{ Hipótesis } & Probabilidad & Tipo de Tendencia \\
\hline 1 & $\begin{array}{l}\text { Si los artesanos continúan con el tradicionalismo } \\
\text { administrativo, se afirma que para } 2027 \text { estas em- } \\
\text { presas seguirán sin brindar protección social para } \\
\text { sus trabajadores. }\end{array}$ & $41 \%$ & Improbable \\
\hline 2 & $\begin{array}{l}\text { Si se maneja un buen plan de capacitación y } \\
\text { asesoría externa el gremio artesanal generará un } \\
\text { variado portafolio de productos para el mercado. }\end{array}$ & $52 \%$ & Moderada \\
\hline 3 & $\begin{array}{l}\text { No permitir que la competencia desplace la calidad } \\
\text { del trabajo artesanal generando estrategias que les } \\
\text { permitan afrontar el cambio, y generado un mayor } \\
\text { posicionamiento en el mercado, sin perder la esen- } \\
\text { cia artesanal. }\end{array}$ & $20 \%$ & Improbable \\
\hline 4 & $\begin{array}{l}\text { La asociatividad generará sinergias y desarrollo for- } \\
\text { taleciendo el gremio artesanal al mejorar las relacio- } \\
\text { nes comerciales. }\end{array}$ & $63 \%$ & Fuerte \\
\hline 5 & $\begin{array}{l}\text { Dar continuidad al legado de los artesanos, para } \\
\text { que la praxis sea la escuela donde las nuevas gen- } \\
\text { eraciones continúen con el desarrollo de estas técni- } \\
\text { cas artesanales. }\end{array}$ & $70 \%$ & Fuerte \\
\hline
\end{tabular}

Tabla 3. Cuadro con puntaje más alto de probabilidad

\begin{tabular}{|c|c|c|}
\hline Número & Escenario & Nombre \\
\hline & 111111 & Artesanías de Pasto en la cumbre \\
\hline & 101111 & Artesanías de Pasto sin asociatividad \\
\hline & 111011 & Artesanías de Pasto sin capacitación \\
\hline
\end{tabular}

Tras analizar la información suministrada por el SMIC se encontró como escenario de mayor probabilidad o fuerza que los artesanos den continuidad al legado, para que la praxis sea la escuela donde las nuevas generaciones continúen con el desarrollo de estas técnicas artesanales. A pesar de eso, se considera prioritario implementar un proceso de asociatividad, para consolidar un gremio fuerte, competitivo y que represente un importante renglón de la economía local, departamental y nacional.

\section{La asociatividad como escenario deseado}

La asociatividad es definida como la unión de voluntades y recursos de un grupo de personas en busca de un objetivo común. Es un proceso que exige compromiso, permanencia y disciplina, es trabajar en conjunto para obtener mejores resultados.

Son de gran importancia los modelos de asociatividad como una estrategia para afrontar los principales problemas que sufren las MIPYMES del sector artesanal de Pasto, tales como el acceso a los recursos financieros, la penetración en los mercados locales e internacionales, la innovación y el desarrollo de nuevos productos.

Los actores fundamentales que contribuyen en la asociatividad son: la Alcaldía-Corpocarnaval, la Oficina de turismo, la Casa de la Cultura, el Laboratorio de Artesanos, Zeyki, y Red Urel, entre otras. Estas instituciones pueden ayudar a desarrollar la asociatividad de diferentes formas para los artesanos de Pasto.

\section{Conclusiones}

La intencionalidad del presente trabajo consistió en el reconocimiento de las potencialidades económicas de una actividad en la cual el departamento de Nariño, y, particularmente, el municipio capital Pasto, presenta un asiento importante en el plano nacional, el Barniz de Pasto, según la técnica del enchapado en tamo. Se trata de una actividad ancestral, que, gracias a las habilidades de los artesanos, se 
ha difundido generacionalmente; sin embargo, hoy requiere un apoyo decidido por parte los entes responsables del desarrollo económico y social, para hacerla cada vez más atractiva e incrementar los niveles de competitividad en los mercados actuales.

El proyecto permitió contar con la colaboración de representantes de los cuatro actores sociales responsables, en gran parte, del desarrollo territorial del gremio artesanal en el municipio de Pasto, y quienes, mediante la participación activa y dialógica, permiten adelantar un diagnóstico de la situación actual desde el comportamiento endógeno y exógeno, siguiendo las directrices diseñadas por Michel Godet para puntualizar los diferentes cambios que se pueden generar en el futuro de un gremio tan importante para esta región.

La prospectiva estratégica para el proyecto desarrollado es una gran opción, pues, de manera sencilla, permite conocer una serie de escenarios a los cuales se verá enfrentado el subsector artesanal del barniz de Pasto mediante la técnica del enchapado en tamo, y considerando que el de mayor relevancia es la apuesta a la asociatividad de un gremio que hasta ahora se ha caracterizado por el trabajo individual.

Con el fin de enfrentar los nuevos cambios generados dentro del mundo globalizado, se formula una serie de estrategias para afrontar los nuevos retos. La actividad artesanal es una potencia económica de la región, con gran reconocimiento en todo el país. Las estrategias definidas requieren el diseño de un plan vigía, con el fin de evitar desviaciones que perjudiquen al sector.

Históricamente se ha demostrado que trabajar de manera individualista no permite ser competitivos; se hace necesaria la complementariedad en casi todas las actividades que desarrolla el ser humano. El panel de expertos programado para el desarrollo de la investigación se convirtió en el punto de partida para el reconocimiento de un gremio de tradición en nuestra región, el cual, durante los últimos años, ha ido perdiendo su importancia, debido a la falta de organización. El futuro, visto desde una reprogramación del presente para el presente objeto de investigación, será más beneficioso si para ello se piensa en una agrupación de quienes dependen para su sustento de la actividad artesanal. La capacitación y la educación en nuevas opciones de producción harán que estas actividades sean cada vez más atractivas y competitivas.

\section{Referencias bibliográficas}

Alcadía de Pasto y PUND Colombia. (2011). Pasto, ciudad región. Bases para una política de inclusión productiva para la situación de pobreza y vulnerabilidad. Bogotá: Impresol Ediciones Ltda.

Cámara de Comercio de Pasto. (2000). Importancia Económica del sector artesanal. San Juan de Pasto: Diseño e impresiones papeles y papeles.

Castañeda, Y., Enríquez, L. \& Nieto, D. (2012). Estudio de mercadeo para los productos artesanales del barniz de Pasto bajo la técnica enchapado en tamo. Bogotá: UNAD.

Godet, M. (2010). Caja de herramientas. Recuperado de www.laprospective.com

Godet, M. (2004). Manual de prospectiva estratégica. Paris: Editorial Dunod.

Instituto Cert. (1999). Diagnóstico y planteamiento de poI Íticas para el sector artesanal. Bogotá: Edición Oficina de Comunicaciones.

Instituto Cert. (1999). Diagnóstico y planteamiento de políticas para el sector artesanal. Bogotá: Edición Oficina de Comunicaciones.Interdesing, Colombia. (1994). Memorias, La artesanía en la decoración de interiores. Bogotá: Artesanías de Colombia S.A.

Kothler, P. (1998). Fundamentos de mercadotecnia. México: Prentice Hall.

Lambin, J. J. (1995). Marketing Estratégico, 3ra. Edición. Madrid: Ed. Mc Graw Hill.

Mojica, F. J. (2002). Modelo prospectivo estratégico y taller de apropiación, modulo básico. Bogotá: Universidad Externado de Colombia.

Pineiro, C. Intercambio compesado. Madrid: Mc Graw Hill.

Artesanías de Colombia. (1995). Revista la aldea Humana/Florianópolis. Bogotá: Colombia, 23-25.

La solución artesanal. Revista Colombiana de antropología. 16, 197.

Secretaría de Agricultura y Medio Ambiente de Nariño. (2007). Consolidado agropecuario 2006. San Juan de Pasto: Secretaría de Agricultura.

www.dane.gov.co

www.mincomeercio.gov.co 